\title{
Circuit Level Model of Miniature Circuit Breakers
}

\author{
F. Bizzarri, Senior Member, IEEE, A. Brambilla Member, IEEE, L. Ghezzi and F. Rigamonti
}

\begin{abstract}
We present a black-box model of miniature circuit breakers having a realistic physical background. In compliance with basic physics, engineering know-how and magneto-hydrodynamics arc simulations, two sets of differential-algebraic equations are derived to model air plasma chemical dynamics and its interaction with breaker's physical environment (chamber shape, splitter plates, arc traveling). A numerically efficient and accurate electrical model at the breaker terminals is proposed. Its behaviour is shown by comparing simulations with short circuit experiments. Applications are at system level, including simulation of networks with several breakers, such as multiphase switching transients, selectivity and coordination with fuses, other breakers and surge protection devices.
\end{abstract}

Index Terms-circuit breaker, black-box model, circuit simulation, system level analysis, plasma model, arc root, arc splitting.

\section{INTRODUCTION}

C IRCUIT breakers protect electrical circuits, equipments and people interacting with them. The miniature circuit breakers (MCBs) are widely employed in both residential and industrial low voltage applications $(<1000 \mathrm{~V}$, AC-RMS; $<1500 \mathrm{~V}, \mathrm{DC})$. Despite the very reduced size, MCBs interrupt prospected short-circuit currents of the order of $10 \mathrm{kA}$ in less than half period of the working frequency $(50-60 \mathrm{~Hz})$. During switching transients, the important class of current limiter MCBs manage to dump the current and, in AC regime, to anticipate the natural current zero. A successful current interruption requires the timely dissipation of the energy stored in the circuit that is massively converted into heat in the electric arc that forms inside the switching $\mathrm{MCB}$, as soon as electrical contacts detach.

Formerly resorting to experimental trial and error, modern design of MCBs heavily relies on computational magnetohydro-dynamics (MHD) [1]. The physics of the switching transient is attacked directly by solving in space and time the dynamics of the gaseous atmosphere that develops inside, starting from air quickly ionized and progressively enriched by metals and plastics that ablate due to ohmic heating. This gaseous atmosphere moves, pushed by pressure gradients and Lorentz force. Characteristic modeling features are, inter alia, Navier-Stokes for transport in the fluid/plasma phase, Maxwell for electromagnetism, Boltzmann for radiation with participating media, Fourier and phase transitions in the solid

Federico Bizzarri is with Politecnico di Milano, DEIB, p.za Leonardo da Vinci, n. 32, I20133 Milano, Italy and also with the Advanced Research Center on Electronic Systems for Information and Communication Technologies E. De Castro (ARCES), University of Bologna, 41026 Bologna, Italy. (e-mail: federico.bizzarri@polimi.it).

Angelo Brambilla is with Politecnico di Milano, DEIB, p.za Leonardo da Vinci, n. 32, I20133 Milano, Italy. (e-mail: angelo.brambilla@polimi.it)

Luca Ghezzi and Francesco Rigamonti are with ABB, Viale Dell'Industria 18， I20010 Vittuone, Italy. (e-mail: \{luca.ghezzi, francesco.rigamonti\}@it.abb.com) phase, and plasma as a strongly nonlinear medium. A large set of partial differential equations are discretized by cooperating numerical methods, including finite volumes, edge elements and (nodal) finite elements. Albeit accurate and insightful, MHD simulations are CPU intensive (order of a day for a complete interruption that lasts about $10 \mathrm{~ms}$ ) and thus unfeasible to address large networks and/or for long transients. On the other hand, circuit breaker manufacturers feel the need for simulating the behavior of MCBs as circuital components. There are various examples of such system level studies on the industry side: i) coordination of MCBs with other protective devices, such as fuses; ii) coordination between breakers with different selectivity levels; iii) coordination between electromechanical breakers and ultrafast solid state contactors or breakers; iv) interruption duty estimation for the last pole to clear current in multi-phase short-circuits; v) MCBs coupled with varistors to dissipate reactive power and provide strong current limitation. In these cases, estimates (including quite coarse estimates) of integral electrical quantities, such as current and voltage levels, the Joule integral (also known as $I^{2} t$ ), the tripping time, and the like are sought for. Applications on the industry side call for fast and lite black-box models for the МСB interacting with the embedding circuit [2], [3]. The MCB circuital behavior in response to boundary conditions simultaneously computed at system level will be delivered. The integral voltage and current time histories are not exogenous, viz. imposed a priori, but, rather, they come from a physically based model, as opposed to inserting measured electrical quantities in a network simulator. In fact, in the latter case only specific testing conditions could be retained and internal model consistency would be lost as soon as network conditions deviate from the experimental ones. The breaker is expected to act as a realistic though not necessarily "exact" network component. The focus is on the overall system behavior, and not on the breaker itself as a system made of parts to be designed.

In the case of MCBs, conditions may exist (especially when a breaker is driven to its physical limit) when the interruption capacity depends on low-level details that, as such, cannot enter the high-level, black-box model. This intrinsic indetermination may be profitably investigated by stochastically changing salient parameters, to be taken as stochastic variables instead of sharp numbers, or also with reference to suitably defined scenario analyses. The very nature of the model, which runs efficiently and can be easily implemented in modern circuit simulators, naturally copes with the need for large numbers of simulation repetitions.

The term black-box implies that all "internal" physical quantities (e.g., arc temperature, electron/ion density, mobility, pressure, flow, etc.) are not fully computed. Only the global effect on the whole breaker behavior is retained. In turn, this means that black-box models can not be used to design or 
modify a breaker. Several black-box models were presented in the literature for breakers ranging from low-voltage to highvoltage applications, using vacuum, air or $\mathrm{SF}_{6}$ gas as dielectric [4]-[11]. We dare say that a common shortcoming of traditional models is the poor link between the arc and the surrounding environment. In a MCB, current interruption is obtained thanks to magnetic fields by guiding the arc in a region where it is fragmented by transversal ferromagnetic splitter plates [12]. Electron transitions from the solid phase, i.e, from an anode or a cathode, into the plasma phase are associated to roughly current independent surfacic voltage drops (in the $[10,20] \mathrm{V}$ range per anode-cathode pair, depending on plate material). Consequently, multiplying the number of serial arc roots, by means of for example 10 splitter plates, amounts to sensibly increasing the total arc voltage drop to a value comparable to the supply voltage. From the circuit standpoint, the effect is equivalent to the insertion of a passive load per splitter, which depresses current flow and where electric power is thermally converted and thus extracted from the electrical domain [13], [14]. These very devices are intentionally designed as vehicles to externally act on arc dynamics and thus making breakers different one from another, even though the arc modeling equations may be considered almost the same.

We firstly present an arc model, based on aggregate equations governing the electron/positive-ion pairs forming the air plasma. These equations are developed by starting from and modifying the prey-predator model proposed in [15]. We thus use the geometric, kinematic and magnetic field properties of the MCB structure to link the proposed generic arc model and to obtain an aggregate model at the two electrical terminals. The key aspect is that the plasma model structure may be ported to different types of gaseous circuit breakers from low to high voltage applications. Model parameters should be adapted to the particular gaseous atmosphere and a dedicated model as that proposed in this paper would be needed for the environment, i.e., the physical structure of the breaker in which the arc is created, operates and extinguishes.

\section{BASIC AIR PLASMA MODEL}

Air is assumed to be composed of nitrogen $\left(\mathrm{N}_{2}\right)$ and oxygen $\left(\mathrm{O}_{2}\right)$ only and they are treated as a unique equivalent substance $\left(\mathrm{X}_{2}\right)$. The relative contributions of neutral molecules $\mathrm{X}_{2}$ at $t$ time and $T$ temperature, with reference to the undissociated state, is denoted by $n_{\mathrm{X}_{2}}(t, T)$. An analogous notation is used for neutrals atoms $\mathrm{X}$, free electrons $\mathrm{e}^{-}$and positive ions $\mathrm{X}^{+}$. The superscript ${ }^{*}$ is used to identify quantities at the equilibrium, e.g., $n_{\mathrm{X}_{2}}^{*}(T)$ represents the stationary value of the relative contributions of neutrals molecules $\mathrm{X}_{2}$ at $T$ temperature. In the following, when feasible, the dependency on $t$ and $T$ is dropped for compactness.

Making reference to Fig. 1, we identify two temperature intervals $\mathcal{I}_{1}=\left[T_{0}, T_{1}\right]$ and $\mathcal{I}_{2}=\left[T_{1}, T_{2}\right]$, that are used to derive a simplified model of the air plasma. In the former, $\mathrm{X}_{2}$ dissociates into $\mathrm{X}$ and at $T_{1}$, if the air plasma is at equilibrium, the dissociation is full, i.e., $n_{\mathrm{X}}^{*}\left(T_{1}\right)=2 n_{\mathrm{X}_{2}}^{*}\left(T_{0}\right)$. The unimolecular thermal decomposition $\mathrm{X}_{2} \leftrightarrow 2 \mathrm{X}$ governing dissociation is assumed to be a first order one-step reaction and thus the

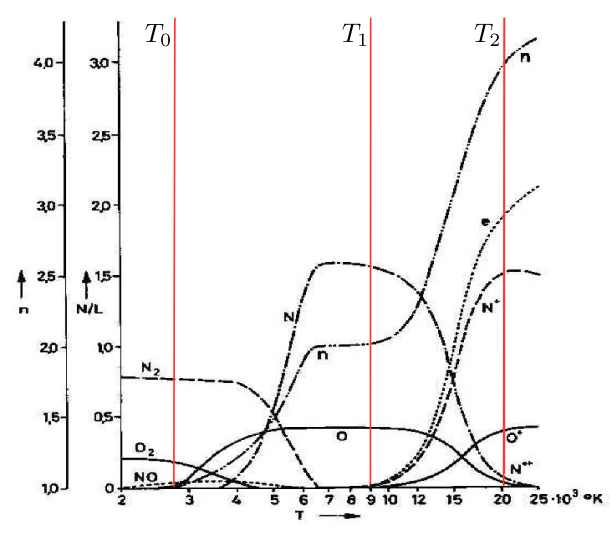

Fig. 1. The dissociation and ionization of air plasma as a function of $T[\mathrm{~K}]$ at $101325 \mathrm{~Pa}$ [16]. $n$ is the total particle number relative to the undissociated state, all other particle species are referred to the $N / L$ scale, expressing their relative contributions, with reference to the undissociated state.

differential form of its rate law is linear. Consequently, the dynamics of $n_{\mathrm{X}}$ can be modeled by the ordinary differential equation (ODE)

$$
\dot{n}_{\mathrm{X}}=\nu\left(n_{\mathrm{X}}^{*}-n_{\mathrm{X}}\right),
$$

where $n_{\mathrm{X}}^{*}$ is prescribed as in Fig. 1 .

First ionization is observed in $\mathcal{I}_{2}$, according to the reaction $\mathrm{X} \rightarrow \mathrm{X}^{+}+\mathrm{e}^{-}$. Since it is assumed that an $\mathrm{X}^{+}-\mathrm{e}^{-}$pair is formed from $\mathrm{X}, n_{\mathrm{X}^{+}}(t, T)=n_{\mathrm{e}^{-}}(t, T)$. The dynamics of $n_{\mathrm{e}^{-}}$ is assumed to be governed by the ODE

$$
\dot{n}_{\mathrm{e}^{-}}=a n_{\mathrm{X}}\left(1+b n_{\mathrm{e}^{-}}\right)-k n_{\mathrm{e}^{-}} n_{\mathrm{X}^{+}}
$$

where the $a, b$ and $k$ parameters are positive. In (2), the $a n_{\mathrm{X}}$ term means that the rate of ionization is proportional to the concentration of neutral atoms, viz. abundance of $n_{\mathrm{X}}$ promotes the creation of $\mathrm{X}^{+}-\mathrm{e}^{-}$pairs. The $-k n_{\mathrm{e}^{-}} n_{\mathrm{X}}+$ term stands for recombination. If $b=0$, at stationary equilibrium $a n_{\mathrm{X}}^{*}=k n_{\mathrm{e}^{-}}^{*} n_{\mathrm{X}^{+}}^{*}=k\left(n_{\mathrm{e}^{-}}^{*}\right)^{2}$, according to basic rules of chemical equilibrium. The $b n_{\mathrm{X}} n_{\mathrm{e}^{-}}$term is introduced since, when several free electrons are present with a significant velocity, they can hit a neutral atom catalyzing the reaction and thus increasing the reaction rate. This phenomenon is proportional to the product of the concentrations. At stationary steady-state,

$$
n_{\mathrm{X}}^{*}+n_{\mathrm{e}^{-}}^{*}=n_{\mathrm{X}}^{*}\left(T_{1}\right)=2 n_{\mathrm{X}_{2}}^{*}\left(T_{0}\right) \equiv \Xi
$$

but, in transient evolution, if one assumes to vary $T$ sufficiently fast, disequilibrium is expected in (3). For this reason it is necessary to introduce a further ODE to describe the dynamical process that guarantees (3). The same consideration can be done concerning $\mathcal{I}_{1}$ in which $n_{\mathrm{e}^{-}}^{*}=0$ at steady state but $n_{\mathrm{e}^{-}} \neq 0$ in transient. Since actually the above mentioned disequilibrium conditions are significant at $T \approx T_{1}$, (1) is used also in $\mathcal{I}_{2}$ assuming that (3) holds, and (2) is extended in $\mathcal{I}_{1}$ by properly acting on its parameters in order to guarantee $n_{\mathrm{e}^{-}}^{*}=0$ for $T \in \mathcal{I}_{1}$. In particular, the $a$ parameter is used to set $n_{\mathrm{e}^{-}}^{*}$, specifically by choosing

$$
a(T)=\frac{k\left(n_{\mathrm{e}^{-}}^{*}\right)^{2}}{\left(1+b(T) n_{\mathrm{e}^{-}}^{*}\right)\left(\Xi-n_{\mathrm{e}^{-}}^{*}\right)},
$$


and $b(T)=b \chi_{\left[T_{1}, T_{2}\right]}(T)$, where $\chi_{\left[T_{1}, T_{2}\right]}(T)$ is the indicator function of the $\left[T_{1}, T_{2}\right]$ interval ${ }^{1}$. The ODEs describing the overall air plasma model for $T \in \mathcal{I}_{1} \cup \mathcal{I}_{2}$ are the following

$$
\left\{\begin{array}{l}
\dot{n}_{\mathrm{X}}=\nu\left(n_{\mathrm{X}}^{*}-n_{\mathrm{X}}\right) \\
\dot{n}_{\mathrm{e}^{-}}=k\left[\frac{\left(1+b(T) n_{\mathrm{e}^{-}}\right)\left(n_{\mathrm{e}^{-}}^{*}\right)^{2} n_{\mathrm{X}}}{\left(1+b(T) n_{\mathrm{e}^{-}}^{*}\right)\left(\Xi-n_{\mathrm{e}^{-}}^{*}\right)}-n_{\mathrm{e}^{-}}^{2}\right] .
\end{array}\right.
$$

For $\quad T \in \mathcal{I}_{2}, \quad$ (5) admits two equilibria, namely $P_{1}=\left[\Xi-n_{\mathrm{e}^{-}}^{*}, n_{\mathrm{e}^{-}}^{*}\right]^{\mathrm{T}}$ and $P_{2}=\left[\Xi-n_{\mathrm{e}^{-}}^{*},-\frac{n_{\mathrm{e}^{-}}^{*}}{1+b n_{\mathrm{e}^{-}}^{*}}\right]^{\mathrm{T}}$. The eigenvalues of the Jacobian matrix computed at $P_{1}$ are $\lambda_{1,1}=-\nu$ and $\lambda_{1,2}=-\frac{k n_{\mathrm{e}^{-}}^{*}\left(2+b n_{\mathrm{e}^{-}}^{*}\right)}{1+b n_{\mathrm{e}^{-}}^{*}}$, whereas at $P_{2}$ are $\lambda_{2,1}=\lambda_{1,1}$ and $\lambda_{2,2}=-\lambda_{1,2}$. Since $k$ and $\nu$ are positive and $b \geq 0, P_{1}$ and $P_{2}$ are a stable and an unstable node, respectively. It is worth noticing that $P_{2}$ is generically not admissible for $T>T_{1}$. At $T_{1}, n_{\mathrm{e}^{-}}^{*}=0$ and consequently, $P_{1}=P_{2}$ and they degenerate in the equilibrium $P_{0}=[\Xi, 0]$, which is non-hyperbolic being $\lambda_{2,2}=\lambda_{1,2}=0$. Despite the fact that $b(T)$ is discontinuous in $T_{1}$, (5) admits a single non-hyperbolic equilibrium for $T \in \mathcal{I}_{1}$ which coincides with $P_{0}$ at $T_{1}$. For this reason it will be referred to as $P_{0}$ also for $T<T_{1} . P_{0}$ turns out to be attractive for $n_{\mathrm{e}^{-}}>0$ and repulsive for $n_{\mathrm{e}^{-}}<0$. Actually this is not an issue since a negative $n_{\mathrm{e}^{-}}$is physically meaningless but it could be a problem from a numerical standpoint. For this reason, for $T \in \mathcal{I}_{1}$, the ODE governing the dynamics of $\mathrm{e}^{-}$is rewritten as $\dot{n}_{\mathrm{e}^{-}}=-k\left|n_{\mathrm{e}^{-}}\right| n_{\mathrm{e}^{-}}$, which makes $P_{0}$ attractive also for $n_{\mathrm{e}^{-}}<0$.

The analysis of the equilibria of (5) is important to guarantee that, either for a fixed $T$ or varying it as an external input for (5), the evolution of $n_{\mathrm{X}}$ and $n_{\mathrm{e}^{-}}$is the expected one.

We chose to model the curves reported in Fig. 1 as

$$
\begin{aligned}
& 2 n_{\mathrm{X}}^{*}(T)=\Xi\left(1+\tanh \left(\gamma_{\mathrm{X}}\left(T-\bar{T}_{01}\right)\right)\right)-2 n_{\mathrm{e}^{-}}^{*}(T) \\
& 2 n_{\mathrm{e}^{-}}^{*}(T)=\Xi\left(1+\tanh \left(\gamma_{\mathrm{e}^{-}}\left(T-\bar{T}_{12}\right)\right)\right),
\end{aligned}
$$

where $2 \bar{T}_{01}=T_{0}+T_{1}$ and $2 \bar{T}_{12}=T_{1}+T_{2}$, while $\gamma_{\mathrm{X}}$ and $\gamma_{\mathrm{e}^{-}}$ are fitting parameters.

\section{THE MCB BLACK-BOX MODEL}

The plasma in (5) is supplemented by the interactions with the electrical quantities and power exchange ruling temperature. The external mechanical actions at contact opening and cooling systems need also be considered. Contacts are assumed to separate with constant speed. Governing equations are gathered into the differential-algebraic system

$$
F(v(t), i(t), x(t), p(t), t)=0,
$$

\footnotetext{
${ }^{1}$ The $\chi_{\mathcal{I}}(x)$ indicator or characteristic function of the generic $\mathcal{I}$ interval is such that $\chi_{\mathcal{I}}(x)=1$ if $x \in \mathcal{I}$ and $\chi_{\mathcal{I}}(x)=0$ if $x \notin \mathcal{I}$.
}

where $F(v(t), i(t), x(t), p(t), t)=$

$$
\left\{\begin{array}{l}
\dot{n}_{\mathrm{X}}-\nu\left(n_{\mathrm{X}}^{*}(T)-n_{\mathrm{X}}\right) \\
\dot{n}_{\mathrm{e}^{-}}-k\left[\frac{\left(1+b(T) n_{\mathrm{e}^{-}}\right)\left(n_{\mathrm{e}^{-}}^{*}(T)\right)^{2} n_{\mathrm{X}}}{\left(1+b(T) n_{\mathrm{e}^{-}}^{*}(T)\right)\left(\Xi-n_{\mathrm{e}^{-}}^{*}(T)\right)}-n_{\mathrm{e}^{-}}^{2}\right] \\
\zeta \dot{T}-\left[v(t) i(t)-\gamma_{r}\left(T-T_{A}\right)^{4}-\gamma_{c} g(t)\left(T-T_{A}\right)+p(t)\right] . \\
i(t)-\frac{1 / 2\left(1+\tanh \left(\gamma_{s}\left(T-T_{1}\right)\right)\right) \sigma n_{\mathrm{e}^{-}}+g(t)}{x_{0}+x(t)} v(t) \\
g(t)-g_{M}\left[1-\frac{t-t_{0}}{\Delta t_{s}} \chi_{\left[t_{0}, t_{0}+\Delta t_{s}\right]}(t)\right] \chi_{\left(-\infty, t_{0}+\Delta t_{s}\right]}(t) .
\end{array} .\right.
$$

The first two equations in (7-8) replicate the air plasma model (5). The third equation in (7-8) governs the dynamics of temperature of the air plasma model. Temperature is viewed as an indicator of the average kinetic energy of the plasma phase and consequently its time derivative is proportional to the power balance of the system. The $v(t) i(t)$ product is ohmic heating power, the $\gamma_{r}\left(T-T_{A}\right)^{4}$ term is a net emission coefficient (NEC) model [17] of the power radiated by the $\operatorname{arc}^{2}, T_{A}$ is the working temperature of the air within the breaker when it is closed. The $\gamma_{c} g(t)\left(T-T_{A}\right)$ term is an expedient with no direct physical meaning, used to control the arc ignition phase by fictitiously lowering the arc temperature just after arc ignition. Full ionization temperature is assumed just when the contacts start separation, i.e., $T\left(t_{0}\right)=T_{2}$. This is a "numerical tricky ignition" of the arc which is necessary since $n_{\mathrm{X}}\left(T_{A}\right)=0$ and thus it is not possible to generate any $\mathrm{X}^{+}-\mathrm{e}^{-}$pair when the contacts start separation. A way to have free $\mathrm{X}^{+}-\mathrm{e}^{-}$pairs is to artificially increase temperature above $T_{1}$ when the metal contacts are closed $\left(t<t_{0}\right)$. Finally, the role of the $p(t)$ function will be clarified in the sequel in this section. The fourth equation in (7-8) is a generalized Ohm's law that models the current flowing through the breaker. The $x_{0}+x(t)$ term takes into account the length of the arc. Both $x_{0}$ and the $x(t)$ function will be detailed once more in the sequel of this section. For the sake of convenience, we define two contributions to arc conductance, that is, $1 / 2\left(1+\tanh \left(\gamma_{s}\left(T-T_{1}\right)\right)\right) \sigma n_{\mathrm{e}^{-}}(t)$, accounting for the number and mobility of free charge carriers in the plasma phase, which falls to 0 when $T \ll T_{1}$, and the numerical expedient $g(t)$. Notice that both are dimensionally a conductance multiplied by a length. $g(t)$ is defined and evolves according to the last equation in (7-8), where $g_{M}$ is the value when the contacts are closed. More precisely, the $g(t)$ contribution to conductance models an ideal opening of the metallic contacts that does not consider any arc. When the metallic contacts of the breaker are closed, the $g(t)=g_{M}$ function dominates $\sigma n_{\mathrm{e}^{-}}(t)$ and gives the main contribution to the conductance of the breaker. During contact separation, which is assumed to have constant speed, the conductance of the metal contacts drops, with $g(t)$ lowering from $g_{M}$ to 0 along the $\Delta t_{s}$ separation time interval. As evident from

\footnotetext{
${ }^{2}$ The $\left(T-T_{A}\right)^{4}$ term is numerically implemented as $\left|T-T_{A}\right|\left(T-T_{A}\right)^{3}$ in order to ensure that when $P_{e}=0, T=T_{A}$ is the unique stable equilibrium point of the ODE governing the dynamics of the air temperature.
} 


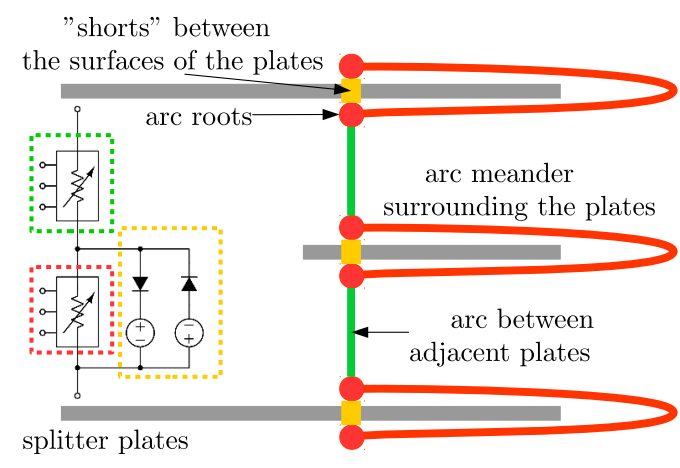

Fig. 2. The physical structure of the MCB used to derive the equivalent circuit of the proposed model.

summing the two contributions to conductance, two resistors are assumed to coexist in parallel connection, they always remain active in the model, and their actual value determines which one practically describes the conductance of the MCB, the other taking negligible values.

In implementing the black-box model of the MCB we considered the interaction between the arc and the environment in which it operates, i.e., the fact that the arc is created between the separating metallic contacts and that it travels towards the splitter, possibly entering it. When the arc enters the splitter after a suitable penetration it is "short circuited" by the metal plates of the splitter, that is, it forms several roots located on the plates. This is schematically represented in Fig. 2, where we have deliberately exaggerated the fact that the arc splits into interplate portions (green) and in meanders (red).

For simplicity, we assumed that the overall arc splits into two equivalent arcs $B_{0}$, representative of all the interplates regions, and $B_{s}$, accounting for all the meanders that are assumed to be simultaneously formed. Two nonlinear elements connected as in Fig. 3 model them. Their electrical behaviour is identical till when the arc enters the splitter.

The plate surrounding portion of the arc increases its length and its voltage till when the voltage drop is high enough to locate an arc root on the plate. The increasing length of the arc surrounding the plates is modeled by the $x(t)$ function in (8). The root location on the splitter plates forces a constant voltage in parallel with $B_{s}$ and the breaker current partitions between the surrounding arc and the path through the plates. The voltage drops due to the arc roots that reside on the splitter plates are modeled through the anti-parallel connection of two branches, each one obtained by connecting in series a diode and a constant voltage source (see Fig. 3). The $i_{r}$ current through $D_{1}$ and $D_{2}$ is different from zero only if $\left|v_{r}\right| \geq v_{d}$, where $v_{d}$ is the same constant voltage of both $E_{1}$ and $E_{2}$. The value of the $v_{d}$ voltage depends on the number of splitter plates. The $D_{1}$ and $D_{2}$ diodes prevent current conduction when the root is not located on the plates. The $v_{r}$ voltage depends on how the arc surrounds the splitter plates, i.e., on the dynamic characteristics of the arc and on the $x(t)$ function.

The cntrl block in Fig. 3 implements the "decisional" actions that the breaker undertakes. The $\left|i_{o}\right|$ current is sensed and when it exceeds an upper threshold the opening process is started (over-current signal). The opening action is sent with

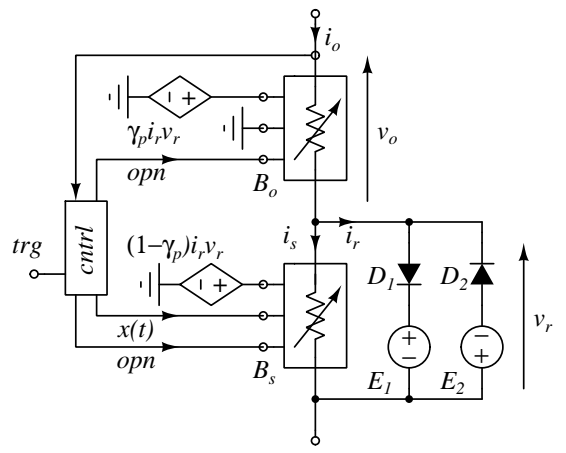

Fig. 3. The schematic of the equivalent circuit of the мсB. Some parts of the model are grouped in the cntrl macro-block. The $B_{o}$ and $B_{s}$ nonlinear elements are identical instances of the same arc model. The $x(t)$ signal drives only $B_{s}$. The $p(t)=i_{r}(t) v_{r}(t)$ instantaneous power drives both $B_{o}$ and $B_{s}$ through two controlled voltage sources.

the $\Delta t_{d}$ delay to $B_{o}$ and $B_{s}$ through the opn pins. The time instant at which these signals are sent is $t_{0}$ in (8). The $\Delta t_{d}$ delay accounts for the mechanical actions that move contacts and that do not act instantaneously when the over-current signal activates the breaker. The movement of the arc that forms between the contacts and is pushed towards the splitter plates is modeled by the second order ODE

$$
\kappa_{1} \ddot{y}(t)+\kappa_{2} \dot{y}(t)-i_{s}^{2}(t) \chi_{\left[t_{0},+\infty\right)}(t)=0
$$

where $\kappa_{1}, \kappa_{2} \in \mathbb{R}$ and $i_{s}(t)$ flows through $B_{s}$. Note that $i_{s}(t)=i_{o}(t)$ till the intervention of the $D_{1}$ or $D_{2}$ diodes. We have assumed that Lorentz force is the main cause that moves the arc towards the splitter plates and that forms the meanders around the plates. The force increases with the square of current flowing through the arc as reported in (9). The $y(t)$ function represents the normalised distance of the traveling arc from the position of the open metal contacts. In a $\mathrm{MCB}$, the arc travels by moving its roots on two almost parallel metal conductors. When $y(t)=1$, it is assumed that the arc touches the splitter plates. The $x(t)$ function governing the length of the arc that surrounds the plates is $x(t)=\kappa_{3}(y(t)-1)^{+}$, where the ${ }^{+}$symbol stands for the positive part of a real.

A further important aspect to be considered is that the two segments of the arc shown in Fig. 2 are heated by the electric power of the roots when the arc enters the splitter. Better said, by considering Fig. 3, when the roots form on the splitter, we have $i_{r}(t) \neq 0$ and $E_{1}$ or $E_{2}$ dissipates the $p(t)=i_{r}(t) v_{r}(t)$ electric power but this would be wrong since, only the arc must dissipate power. The $p(t)$ function is one of the terms of the third equation in (7-8) that models power balance and that we left unexplained. The $p(t)$ power of these branches is thus "re-injected" in the arcs by the controlled sources and dissipated only through the third equation of (7-8). This power is re-injected with different fractions in both $B_{o}$ and $B_{s}$.

The complete black-box model of the MCB that implements 
the $B_{o}$ and $B_{s}$ arcs is

$$
\left\{\begin{array}{l}
F\left(v_{o}, i_{o}, 0, \gamma_{p} p(t), t\right)=0 \\
F\left(v_{r}, i_{s}, x(t),\left(1-\gamma_{p}\right) p(t), t\right)=0 \\
\kappa_{1} \ddot{y}(t)+\kappa_{2} \dot{y}(t)-i_{s}^{2}(t) \chi_{\left[t_{0},+\infty\right)}(t)=0 \\
x(t)-\kappa_{3}(y(t)-1)^{+}=0 \\
i_{x}\left(e^{\alpha\left(v_{r}-v_{d}\right)}-e^{-\alpha\left(v_{r}+v_{d}\right)}\right)-i_{r}=0 \\
i_{o}(t)-i_{s}(t)-i_{r}(t)=0
\end{array}\right.
$$

where the $F(\cdot)$ function is defined by $(7), i_{o}(t)$ is the current through the electrical port and $v_{o}(t)+v_{r}(t)$ is the port voltage, $i_{x}$ is the saturation current of the $D_{1}$ and $D_{2}$ diodes (simple LEVEL1 model of SPICE [18]).

\section{PARAMETER FITTING}

The parameters that are used to fit the proposed model versus experimental data are reported in Table I, with their description, unit and range. These parameters are crucial in accurately reproducing the breaker dynamical behavior. Along with these parameters, the model is characterized by an additional set of coefficients that are not particularly critical and not involved in the optimisation procedure. Their description, unit, value and range are reported in APPENDIX A (see Table III).

The coarse values of the initial guess used in the normalisation and to start the optimisation were determined by hand, through tentative simulations. The optimisation method chosen to fit values of these parameters is the LevenbergMarquardt algorithm embedded in the LSQNONLIN MATLAB function. The objective function (cost function) accounts for a time domain analysis along a suitable time interval during which the MCB opens. This analysis can be performed on a single breaking test or on several tests. It returns a vector whose entries are weighted sums of the differences between the experimental and simulated currents and between the simulated and experimental voltages of the breaker.

The optimisation process can be carried out in cascaded steps and some parameters may be grouped during this process. The suggested optimisation flow is not mandatory and, even though we propose a possible parameter grouping, this does not mean that they act exclusively in the reported time intervals and on the mentioned quantities only. What we suggest may be done at the beginning of the optimisation phase to simplify it and to obtain a satisfactory initial guess for the complete optimisation where all the parameters are involved.

The $\kappa_{1}$ and $\kappa_{2}$ and $\kappa_{3}$ parameters govern the time instant at which the arc engages the splitter. For example this can be identified by the position of the cusp of the current and by the corresponding sudden increase of $v_{o}$ in Fig. 5. An initial optimisation may be carried out to obtain the values of these parameters to have a satisfactory time location of the cusp in the current shape. The $\gamma_{r}, \sigma_{o}, \sigma_{s}$ and $k$ parameters govern

\footnotetext{
${ }^{3}$ In (9) the $y$ variable is dimensionless and the coefficient of proportionality in the relation between the Lorentz force and the square of current is assumed to be unit. Numerical values and units of $\kappa_{1}$ and $\kappa_{2}$ account for these assumptions.
}

TABLE I

FITTING PARAMETERS OF THE MCB

\begin{tabular}{|c|c|c|c|c|}
\hline Name & Description & Unit & Range & Value \\
\hline$v_{d}$ & $\begin{array}{l}\text { Arc voltage drop across all } \\
\text { the splitter plates }\end{array}$ & $\mathrm{V}$ & {$[250,350]$} & 295 \\
\hline$\nu$ & $\begin{array}{l}\text { Natural frequency of the } n_{\mathrm{X}} \\
\text { neutrals atoms dynamics }\end{array}$ & $\mathrm{s}^{-1}$ & {$\left[10^{5}, 10^{7}\right]$} & $10^{6}$ \\
\hline$\gamma_{p}$ & $\begin{array}{c}\text { Parceling out factor of the } p(t) \\
\text { power dissipated by } E_{1}, E_{2} \\
\text { and re-injected in both the } \\
B_{o} \text { and } B_{s} \text { arc models }\end{array}$ & - & {$[0,1]$} & 0.9 \\
\hline$\zeta$ & $\begin{array}{l}\text { Scale factor governing the } \\
\text { evolution of the } T \text { temperature }\end{array}$ & $\frac{\mathrm{sW}}{\mathrm{K}}$ & $(0,1]$ & 0.043 \\
\hline$\sigma_{o}$ & $\begin{array}{l}\text { Mobility of free charge carriers } \\
\text { in the plasma phase for } B_{o}\end{array}$ & Scm & {$[50,350]$} & 298 \\
\hline$\sigma_{s}$ & $\begin{array}{l}\text { Mobility of free charge carriers } \\
\text { in the plasma phase for } B_{s}\end{array}$ & Scm & {$[50,350]$} & 95 \\
\hline$\gamma_{r}$ & $\begin{array}{l}\text { Net emission coefficient of the } \\
\text { arc thermal radiation }\end{array}$ & $\frac{\mathrm{W}}{\mathrm{K}^{4}}$ & {$\left[10^{-9}, 10^{-6}\right]$} & $6 \cdot 10^{-12}$ \\
\hline$k$ & $\begin{array}{l}\text { Weight of the } n_{\mathrm{e}^{-}} n_{\mathrm{X}}+ \\
\text { recombination term in the } \\
n_{\mathrm{e}^{-}} \text {dynamics }\end{array}$ & - & {$[0, \infty)$} & $1.26 \cdot 10^{8}$ \\
\hline$\kappa_{1}$ & $\begin{array}{l}\text { Acceleration }{ }^{3} \text { in the (9) ODE } \\
\text { governing the arc movement }\end{array}$ & $\mathrm{A}^{2} \mathrm{~s}^{2}$ & {$[0, \infty)$} & 4.54 \\
\hline$\kappa_{2}$ & $\begin{array}{l}\text { Friction term in the (9) ODE } \\
\text { governing the arc movement }\end{array}$ & $\mathrm{A}^{2} \mathrm{~s}$ & {$[0, \infty)$} & 2.43 \\
\hline$\kappa_{3}$ & $\begin{array}{l}\text { Coefficient ruling the conversion } \\
\text { (see }(10) \text { ) of the } y \text { normalised } \\
\text { distance of the traveling arc } \\
\text { from the position of the open } \\
\text { metal contacts and the } x \text { length } \\
\text { of the arc surrounding the plates }\end{array}$ & $\mathrm{cm}$ & {$[0, \infty)$} & 19 \\
\hline
\end{tabular}

the shape of the rising current in Fig. 5 (before the cusp). Therefore these parameters and the previous ones allow us to have an initial approximation of the rising current till the time instant at which the arc engages the splitter plates. $\kappa_{3}$, $\gamma_{p}, \sigma_{o}$ and $v_{d}$ govern the current fall down and its shape. They impact also on the duration of the fall phase and up portion of $v_{o}$ visible in Fig. 5 just before the drop at about $68.75 \mathrm{~ms}$. The dynamics of the arc extinction, current zerocrossing and post-arc is mainly governed by $\zeta$ and $k$. Once an initial satisfactory value of the parameters is obtained the complete optimisation with the full set of parameters and possibly several experimental data sets is performed. We used one test case (TS3 of Table II) to perform the initial optimisation and some other test cases to refine it (TS2 and TS4 of Table II). The obtained values are reported in the last column of Table $\mathrm{I}^{4}$.

\section{NUMERICAL AND EXPERIMENTAL RESULTS}

After parameter identification over a subset of available experimental data, the proposed model was tested by comparing its predictions with the experiments and with MHD

\footnotetext{
${ }^{4}$ The parameters are identical for both the $B_{o}$ and $B_{s}$ arc models, but $\sigma$, split as $\sigma_{o}$ and $\sigma_{s}$ in Table I.
} 
TABLE II

Single PhASE TEST CASES (CIRCUIT SCHEMATIC IN Fig. 4)

\begin{tabular}{cccccc} 
Name & Irms & $\cos \boldsymbol{\phi}$ & $\boldsymbol{\theta}$ & $\mathbf{I}_{\mathbf{e}}^{\mathbf{2}} \mathbf{t}$ & $\mathbf{I}_{\mathbf{s}}^{\mathbf{2}} \mathbf{t}$ \\
\hline TS1 & 1500 & 0.93 & $15^{\circ}$ & - & - \\
TS2 & 6000 & 0.65 & $15^{\circ}$ & 39359 & 36255 \\
TS3 & 6000 & 0.65 & $45^{\circ}$ & 44034 & 50086 \\
TS4 & 6000 & 0.65 & $75^{\circ}$ & 42116 & 44337 \\
TS5 & 6000 & 0.65 & $75^{\circ}$ & - & - \\
TS6 & 4500 & 0.75 & $45^{\circ}$ & 34447 & 33088
\end{tabular}

simulations. To this end we replicated the circuit used in experimental tests. The schematic of the single-phase test circuit is shown in Fig. 4. The $R_{o}$ resistance and the $L_{o}$ inductance model the load impedance and set the value of the short circuit current. Their values are set according to short circuit test conditions and are governed by the prospective rms current and $\cos (\phi)$ of the test ${ }^{5}$. The short circuit condition is applied by closing the main $\mathrm{Sw}$ switch.

The test conditions used with this circuit are reported in Table II. The electric angle $\boldsymbol{\theta}$ defines the insertion angle of the short circuit fault, relative to the $e(t)$ supply voltage. More precisely, the $\mathrm{Sw}$ master breaker is initially open, the MCB contacts being closed. Then, $\mathrm{Sw}$ is closed when the $e(t)$ sinusoid is at angle $\theta$ of its cycle (viz., in a suitable and prescribed time instant). $e(t)$ has a peak value of about $230 \sqrt{2} \mathrm{~V}$ (values slightly vary from experiment to experiment) and a period of $20 \mathrm{~ms}(50 \mathrm{~Hz})$. In Table II, the Irms label means the rms value assumed by the prospective current during the short circuit. The $\mathbf{I}_{\mathbf{e}}^{2} \mathbf{t}$ column reports the $i^{2} t$ value computed using the experimental waveforms (average value of a set of experiments) and the $\mathbf{I}_{\mathrm{s}}^{2} \mathrm{t}$ column reports the corresponding ones computed using the simulated waveforms. These values are useful to see how much energy passes through the breaker during the interruption.

All the reported simulations were performed on a LINUXINTEL-I7 $2.3 \mathrm{GHz}$ computer. CPU times never exceeded a few seconds.

In these cases, the MCB is not driven by its current sensing and actuation means but forced externally through the trg terminal in Fig. 3. Better said, during the simulation to exactly apply the prescribed opening time instant we acted on the external trg terminal and blocked the МСв current sensing and actuation means. The simulation and experimental results obtained in the Ts 3 case are compared in Figures 5. In the upper panel of Fig. 5 we report the simulated current (red) and a set of experimental ones (gray region) by the same breaker. We underline that the same breaker operated in the

\footnotetext{
${ }^{5}$ The prospective current is the current that will flow in the circuit if the МСв is kept closed, i.e., it does not interrupt the circuit current.
}

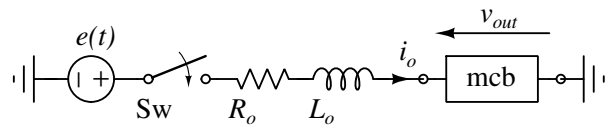

Fig. 4. The schematic of the single-phase test circuit. Sw is the master switch and $v_{\text {out }}=v_{o}+v_{r}$ (see Fig. 3).

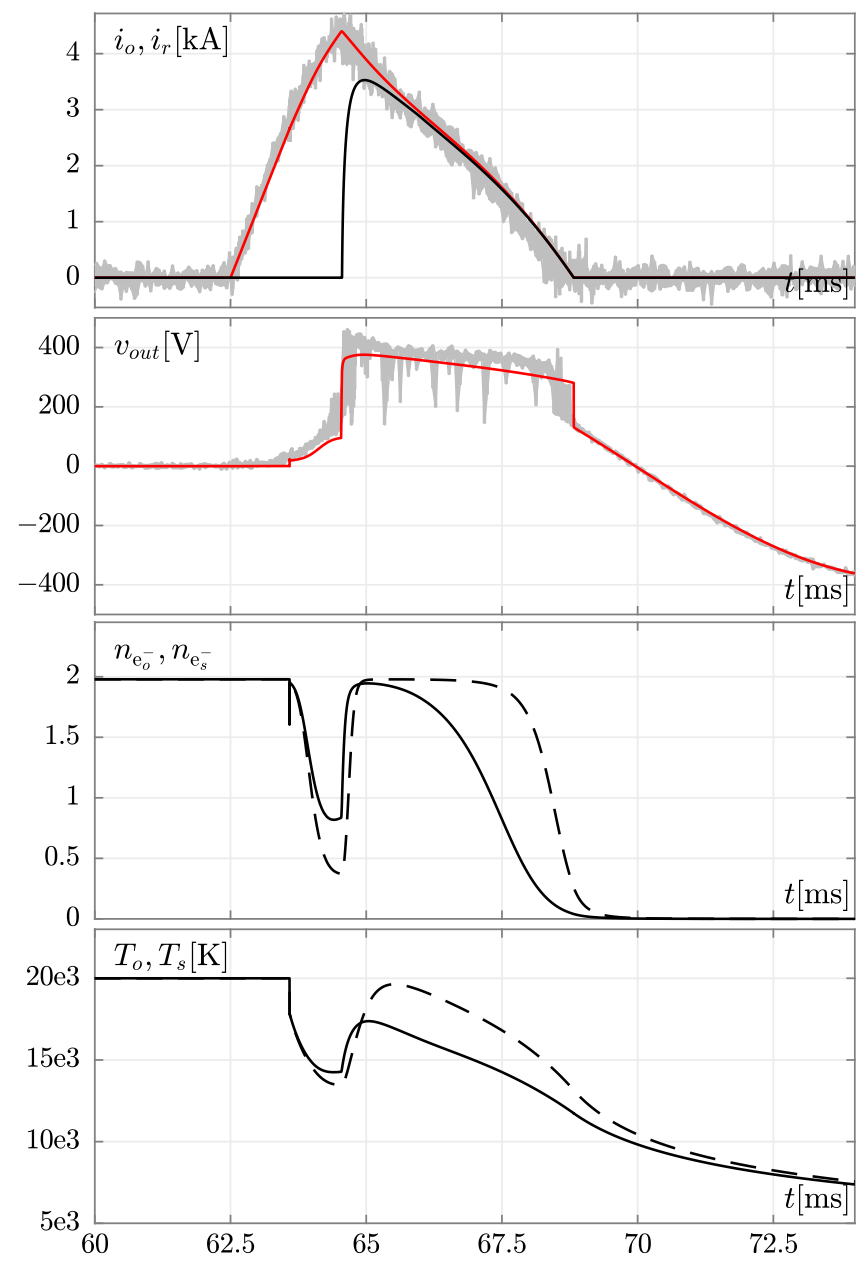

Fig. 5. The $i_{o}$ current through the breaker during the Ts 3 test. $\mathrm{x}$-axis: time [ms], y-axis: experimental (gray) and simulated (red) current [kA]. The gray region represents variability of behavior of the same breaker operated in the same test circuit in different repetitions of the experiment. The $v_{o u t}=v_{o}+v_{r}$ voltage through the breaker; y-axis: experimental (gray) and simulated (red) [V]. Some internal variables of the model. Upper panel: the $i_{o}$ and $i_{r}$ currents [kA], center panel: the $n_{\mathrm{e}^{-}}$of $B_{o}$ (dashed curve) and $B_{s}$ (solid curve), lower panel: the $T$ temperatures of $B_{o}$ (dashed curve) and $B_{s}$ (solid curve) [K].

same test circuit gives similar but not identical current and voltage waveforms from test to test. Variability is an intrinsic aspect of arc behaviour and may be large when breakers are still under development. A clear pictures of this aspect is shown in Fig. 5, where the gray regions refer to different behaviors of a breaker of the same type operated in the same test circuit and conditions. It is immediate to appreciate how current and voltage spread originating the gray regions. A very good behaviour of the proposed model can be appreciated both during the current rising portion of the waveform and during the falling portion, where the arc should be inside the splitter plates, limiting current and dissipating energy.

In the panel just below the upper one of Fig. 5 we report the simulated (red) and experimental (gray) arc voltages. The arc voltage suddenly increases when the arc enters the splitter plates, it varies with a realistic shape during the current limiting interval and suddenly drops when the arc extinguishes. After this drop the arc voltage follows $e(t)$. In Fig. 5 we show 
also some internal variables such as $T, n_{\mathrm{e}^{-}}$and $i_{r}$. When the $i_{o}$ current starts to increase at about $62.5 \mathrm{~ms}$, just after the application of the short circuit, the $T$ temperatures of $B_{o}$ and $B_{s}$ remain fixed at $20000 \mathrm{~K}$ till about $63.5 \mathrm{~ms}$, that is, for the $\Delta t_{d}$ time interval (see Table III). As already said, this time interval is due to the mechanical delay in the contact opening. After $\Delta t_{d}$ temperatures fall in a similar way in both $B_{o}$ and $B_{s}$ and so do the $n_{\mathrm{e}^{-}}$concentrations. Recall that during this phase the splitter is not engaged and the unique breaker arc is modeled as two arcs "connected in series". When the arc engages the splitter plates, the $i_{r}$ current starts to flow across them, i.e., the current flows across $E_{1}$ or $E_{2}$. Its shape mainly governs that of the external $i_{o}$ current since the one across the meanders, i.e., $B_{s}$, largely reduces. The temperature of the plasma between the splitter plates increases from about $14000 \mathrm{~K}$ to about $20000 \mathrm{~K}$ since the current limiting, i.e., energy dissipation is almost completely carried out by the roots of the arcs on the splitter plates. This is the phase that limits the current flowing through the breaker and that extinguishes the current prematurely with respect to the perspective current zero. Voltage drop is practically entirely located on the arc roots while the plasma between the splitter plates is a good conductor. This is well modeled by the increase of the $T$ temperature in $B_{o}$. This causes also the increases of the $n_{\mathrm{e}^{-}}$ concentration of $B_{o}$ that saturates in about $2 \mathrm{~ms}$ and then fully vanishes in a slightly longer time period.

One can conclude that the simulation results are very good since the model was fitted on these very experimental data. We underline that results are good since the proposed model is good enough to almost perfectly fit experimental data. The key aspect is that its behaviour remains acceptable also in other different working conditions.

The currents and voltages of the TS 2 test case are shown in Fig. 6. By comparing the peak value of the short circuit current in the upper panel of Fig. 6 to that of Fig. 5, we see that the former is remarkably less. A good agreement similar

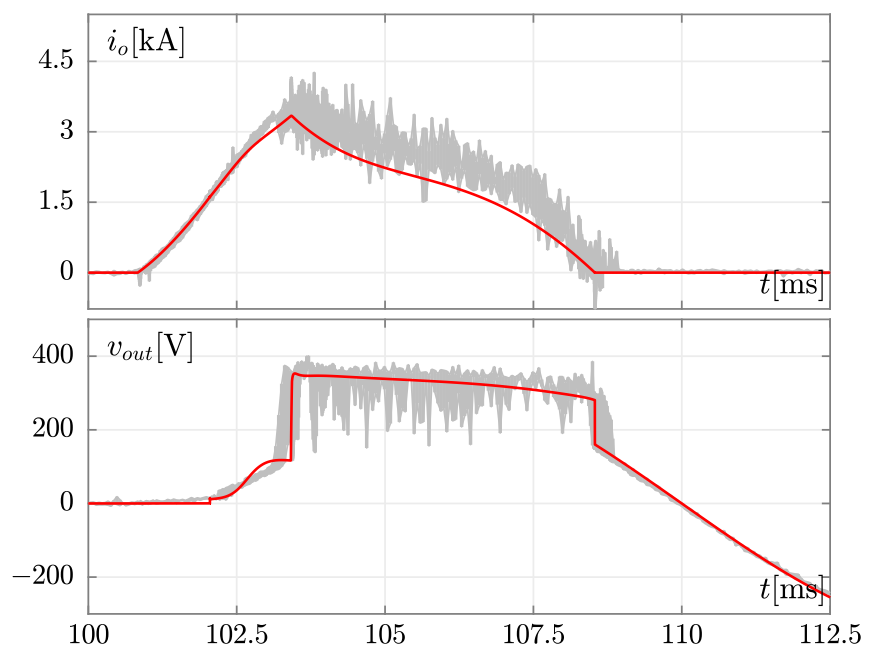

Fig. 6. The $i_{o}$ current through the breaker during the TS2 test. X-axis: time [ms], y-axis: experimental (gray) and simulated (red) current [kA]. The $v_{\text {out }}=v_{o}+v_{r}$ voltage through the breaker during the TS2 test. $\mathrm{x}$-axis: time [ms], y-axis: experimental (gray) and simulated (red) voltage [V]. to that of TS3 is found in short circuit current, limiting action and arc voltage shape. This case shows that the proposed MCB model is candidate to work in a satisfactory way in a large set of working conditions. The good behaviour of the model can be seen also for the TS4 test case. Simulation and experimental results are compared in Fig. 7.

Fig. 8 shows the results of the TS 6 test. The main aspect is that the parameters of the test circuit were varied to obtain a perspective current of $4.5 \mathrm{kA}$ with respect to the $6 \mathrm{kA}$ of the previous cases. Once more we can appreciate the very good agreement between the simulation and experimental results.

The results of the TS5 test are reported in Fig. 9. This is a "bad" test case; it refers to working conditions identical to TS4, but shows an inadequate behaviour of the proposed model. The simulated current is much more limited than the experimental one and crosses zero largely before. The peak value of the short circuit current is the highest of the reported tests since $\theta$ has a value close to $90^{\circ}$. By observing the experimental arc voltage in the lower panel of Fig. 9, we see that the arc enters the splitter as does the simulated arc but the experimental one exits the splitter and does not enter anymore. The entering/exiting the splitter can be inferred by observing the arc voltage. When the arc enters the splitter its voltage suddenly increases since several arc roots locate on the splitter (about $20 \mathrm{~V}$ per root). The opposite happens when the arc exits the splitter. Arc entering/exiting the splitter is a very complex phenomenon governed by several effects, among these by pressure. The arc voltage drops when the arc exits the splitter and thus the experimental short circuit current is less limited and crosses zero at a time instant close to the prospective current. In the proposed model we do not take into account the possibility that the arc exits the splitter after it entered if current does not cross zero. Thus in the simulation when the arc enters the splitter plates in the high current regime it stays inside. This is clearly manifested by the simulated arc voltage that is greater than the experimental one

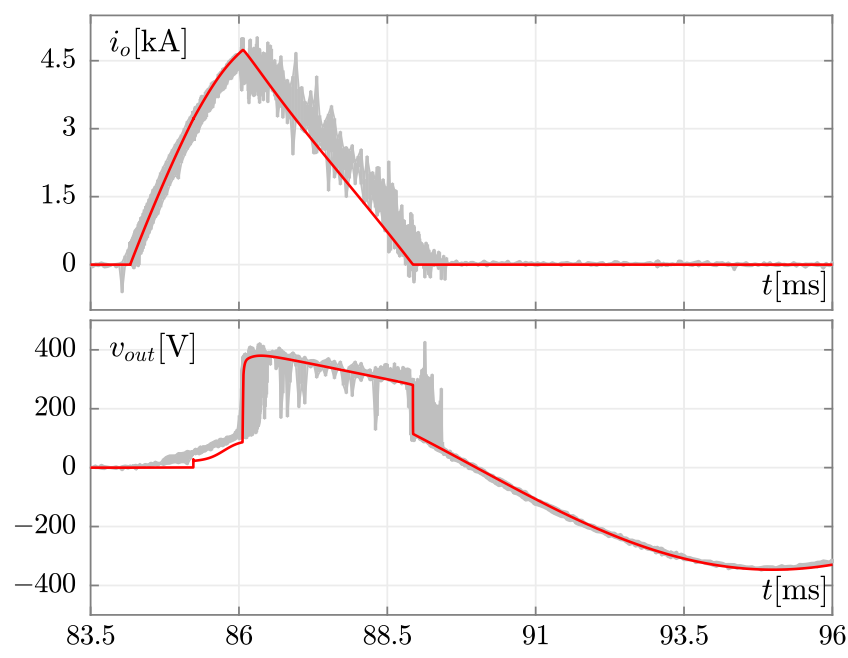

Fig. 7. The $i_{o}$ current through the breaker during the TS4 test. X-axis: time [ms], y-axis: experimental (gray) and simulated (red) current [kA]. The $v_{\text {out }}=v_{o}+v_{r}$ voltage through the breaker during the TS4 test. X-axis: time [ms], y-axis: experimental (gray) and simulated (red) voltage [V]. 
and drops before it, due to the higher current limiting action.

We remark that the possible arc entering/exiting the splitter is intentionally left out of the scope of the black-box model, and that this is not a limitation but, rather, recognizing the right role of this tool. A different analysis (such as MHD simulation), with a way deeper detail level, should be used to address MCB internal design, while black-box modeling will profitably simulate the behavior of an already well designed MCB in a system level study, focused on network related problems, such as selectivity, coordination and the electrical boundary conditions over the last pole to open in a multi-phase interruptions. The (frequently randomic) role played by factors like arc entering/exiting the splitter must remain exogenous, and may possibly be accounted for in system level simulations by means of, e.g., Monte Carlo analyses or scenario analyses.

Finally, we considered the TS1 single-phase test which is at the lowest short circuit current of the set. There are two breaker poles connected in series, interrupting phase and neutral. The neutral pole has a simpler physical structure; for example it may lack the splitter. When its metal contacts separate, the arc burns generating a low voltage drop until the current is interrupted mainly by the phase pole. In the simulation, the experimental voltage of the neutral breaker is replicated by an independent voltage source connected in series to $e(t)$.

The results are reported in Fig. 10. In this case the arc does not largely engage the splitter. This can be seen from the experimental voltage waveform in the lower panel of Fig. 10.

To show a possible application of a black-box model of a $\mathrm{MCB}$, consider the circuit shown in Fig. 11. It implements a three phase interruption circuit that uses a three poles plus neutral MCB. $e_{a}(t), e_{b}(t)$ and $e_{c}(t)$ form a three-phase generator where each has the $230 \sqrt{2} \mathrm{~V}$ peak value referred to ground. As in the previous single phase test circuit resistors and inductors are set according to the short circuit current of $6000 \mathrm{~A}$ with $\cos (\phi)=0.65$ and $\theta=45^{\circ}$, i.e, the same conditions as the TS3 single-phase test. The opening of the

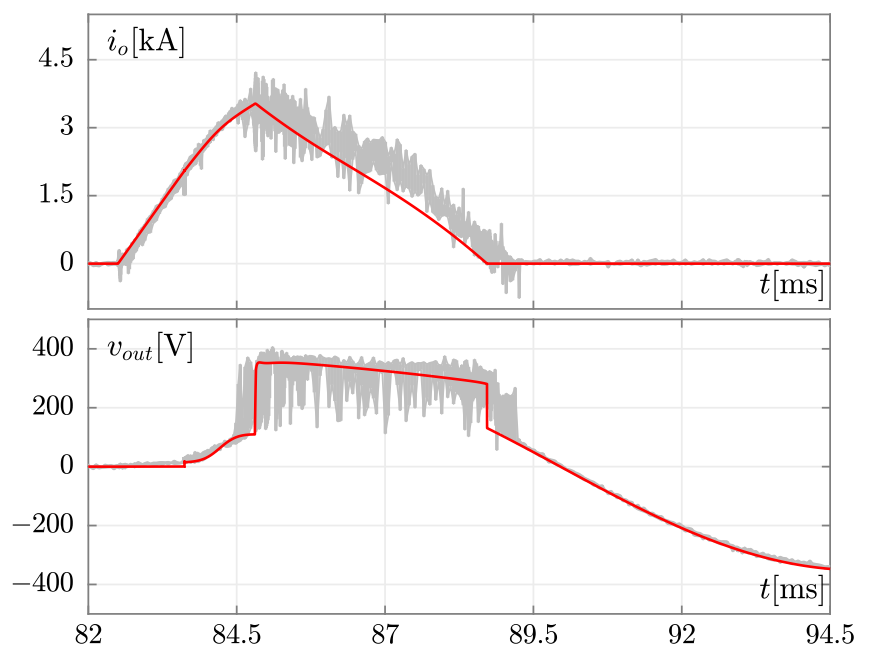

Fig. 8. The $i_{o}$ current through the breaker during the TS6 test. X-axis: time [ms], y-axis: experimental (gray) and simulated (red) current [kA]. The $v_{\text {out }}=v_{o}+v_{r}$ voltage through the breaker during the TS6 test. $\mathrm{y}$-axis: experimental (gray) and simulated (red) voltage [V]. neutral represents a typical pole coordination problem. The neutral pole is closed before the three-phase ones to exploit zero-current operation. Similarly we expect that it has to be the last one to open to exploit once more zero current during a short circuit. To check this we performed two different simulations: a) opening the neutral synchronously with the three phase poles and b) by delaying its opening by $6 \mathrm{~ms}$, i.e., a very long time interval after which the short circuit should be cleared. Note that the neutral pole does not have the splitter. Breaker tripping is triggered by the current of the first phase that goes above $200 \mathrm{~A}$ threshold. The opening signal is simultaneously sent to all phases after $\Delta t_{s}$.

The current of the three phases and neutral of these two cases are shown in Fig. 12. We immediately see that the fastest interruption is obtained by not delaying the opening of the neutral. For case a) the sum of the $\mathrm{I}^{2} \mathrm{t}$ of each phase is 64888 , interruption lasts about $5 \mathrm{~ms}$ and the current through the neutral has a peak value of $140 \mathrm{~A}$. For case b), $\mathrm{I}^{2} \mathrm{t}=81644$, the interruption lasts $7.35 \mathrm{~ms}$ and the neutral peak current is $2900 \mathrm{~A}$. This happens since in case b) the three phases open in an uncoordinated way while in case a) the first pole to (almost) open is " $c$ ", the breaker starts to work in the "two-phase" mode and then clears the short circuit.

\section{CONCLUSIONS}

We have presented a new black-box model for miniature circuit breakers that can be efficiently used to adequately and accurately simulate a complex current interruption process involving several MCBs. The model has physical sound and is based on a completely different approach with respect to well known and celebrated black-box models reported in the literature such as for example the Mayr, Cassie, Urbanek, Schwarz ones.

The effectiveness of the proposed model is shown by comparing simulation results to experimental data obtained from a conventional set of short circuit tests. The proposed model

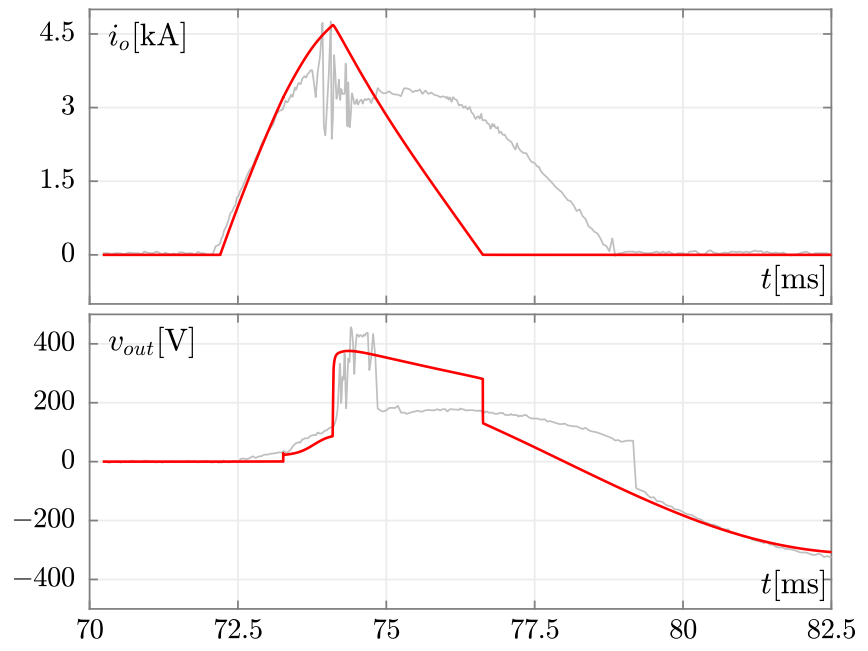

Fig. 9. The $i_{o}$ current through the breaker during the TS5 test. x-axis: time [ms], y-axis: experimental (gray) and simulated (red) current [kA]. The $v_{\text {out }}=v_{o}+v_{r}$ voltage through the breaker during the TS5 test. $\mathrm{y}$-axis: experimental (gray) and simulated (red) voltage [V]. 
is intended to describe an MCB's behavior at system level, and not for the internal design of a MCB. It is not guaranteed that the training set of data used for parameter identification is sufficient to completely characterize any possible working condition.

Of the complex arc physics, some aspects remain out of the proposed model. Among those we recall dielectric breakdown [19], [20], which seems not to affect the kind of circuit breakers at hand. Other important features remain intentionally out of scope, even though they are known to play a crucial role in current interruption processes. For instance, in our model the arc cannot leave the splitter plates once it has entered at high current levels. This means that restrike phenomena (i.e., the arc re-ignites out of the splitter plate region), which are known to occur in circuit breakers, are not within the modeling capabilities. We also assume that the arc either crosses all or none of the splitter plates, though it is known that in real circuit breakers only a portion of available plates may be engaged by the arc, at least in some period during the interruption process. Introducing said features would imply either increasing the level of detail, as MHD models do, or introducing a large plurality of parameters, with obvious over-fitting risks. Both ways would not comply with black-box modeling philosophy and its motivating goal in industry-level, $\mathrm{MCB}$ engineering.

\section{APPENDIX A}

The value of the $T_{0}, T_{1}$ and $T_{2}$ parameters in Table III can be derived from Fig. 1 and may be considered "typical" for air. $T_{A}$ is set at ambient temperature. In principle this is not correct, but the arc is surely completely extinguished when $T<T_{0}$ and an error of a few tens of degree in the third equation of (8) is largely tolerable. The value of $\Xi$ is implicit to the proposed model since when neutrals dissociate they generate an equal number of electrons and positive ions, thus "population" doubles. The values of $\gamma_{\mathrm{e}^{-}}$and $\gamma_{X}$ lead to a complete state change with a variation of a few thousands

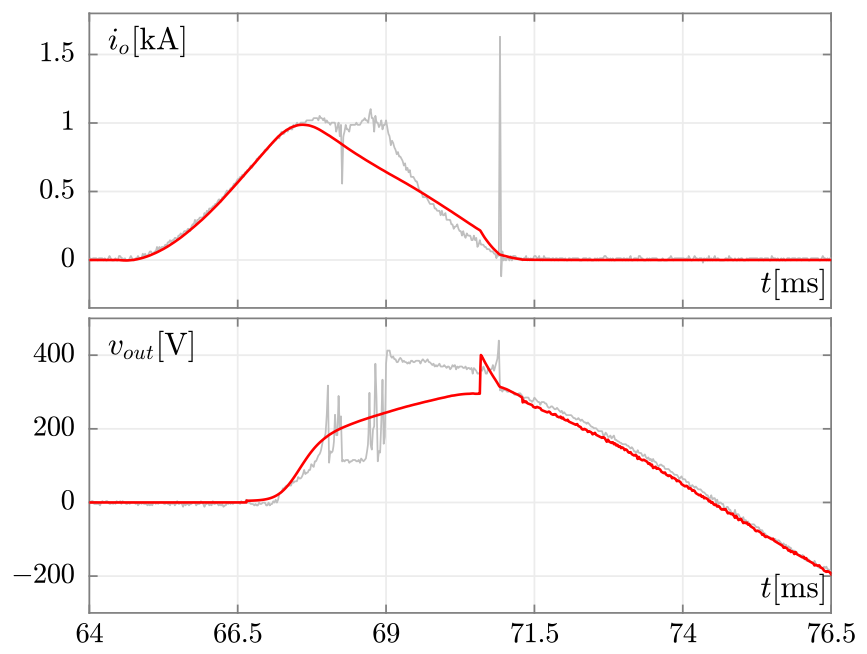

Fig. 10. The $i_{O}$ current through the breaker during the TS1 test. X-axis: time [ms], y-axis: experimental (gray) and simulated (red) current [kA]. The $v_{\text {out }}=v_{o}+v_{r}$ voltage through the breaker during the TS1 test. $\mathrm{y}$-axis: experimental (gray) and simulated (red) voltage [V].

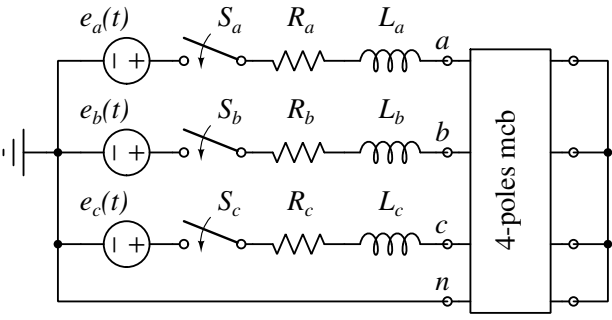

Fig. 11. The schematic of the three-phase, plus neutral test circuit.

TABLE III

FIXED PARAMETERS OF THE MCB USED IN TEST CIRCUITS

\begin{tabular}{ccc|ccc} 
Name & Unit & Value & Name & Unit & Value \\
\hline \hline$T_{0}$ & $\mathrm{~K}$ & $6.5 \cdot 10^{3}$ & $T_{1}$ & $\mathrm{~K}$ & $9 \cdot 10^{3}$ \\
$T_{2}$ & $\mathrm{~K}$ & $2 \cdot 10^{4}$ & $T_{A}$ & $\mathrm{~K}$ & 300 \\
$\Xi$ & - & 2 & $b$ & - & 0.1 \\
$\gamma_{\mathrm{e}^{-}}$ & - & $7 \cdot 10^{-4}$ & $\gamma_{s}$ & $\mathrm{~K}^{-1}$ & 1.66 \\
$\gamma_{X}$ & - & $7 \cdot 10^{-4}$ & $i_{x}$ & $\mathrm{~A}$ & $10^{-12}$ \\
$\alpha$ & $\mathrm{V}^{-1}$ & 40 & $g_{M}$ & $\mathrm{Scm}$ & $10^{4}$ \\
$\Delta t_{s}$ & $\mathrm{~s}$ & $10^{-6}$ & $\Delta t_{d}$ & $\mathrm{~s}$ & $10^{-3}$ \\
$x_{o}$ & $\mathrm{~cm}$ & 1 & $\gamma_{c}$ & $\mathrm{~W}[\mathrm{SKcm}]^{-1}$ & 1
\end{tabular}

Kelvin of the $T$ temperature. This is coherent with Fig. 1. The value of $\gamma_{s}$ is not critical, it contributes to the numerical "trick" used to artificially ignite the arc. It is selected by hand to ensure an adequate temperature drop in the very first phase of arc ignition (see Fig. 5). The $i_{s}$ current is not critical and governs the voltage across the diodes. Note that the current characteristic of the diode is exponential and thus extremely large currents lead to a voltage drop of a few volts. This value is well below $v_{d}$ (the voltage drop across all the splitter plates). $\alpha$ is an approximate value of the voltage equivalent of temperature. $g_{M}$ can be derived considering the resistance of the closed metallic contacts of the breaker. $\Delta t_{d}$ is derived from the MCB technical documentation. $\delta t_{s}$ models the time spent

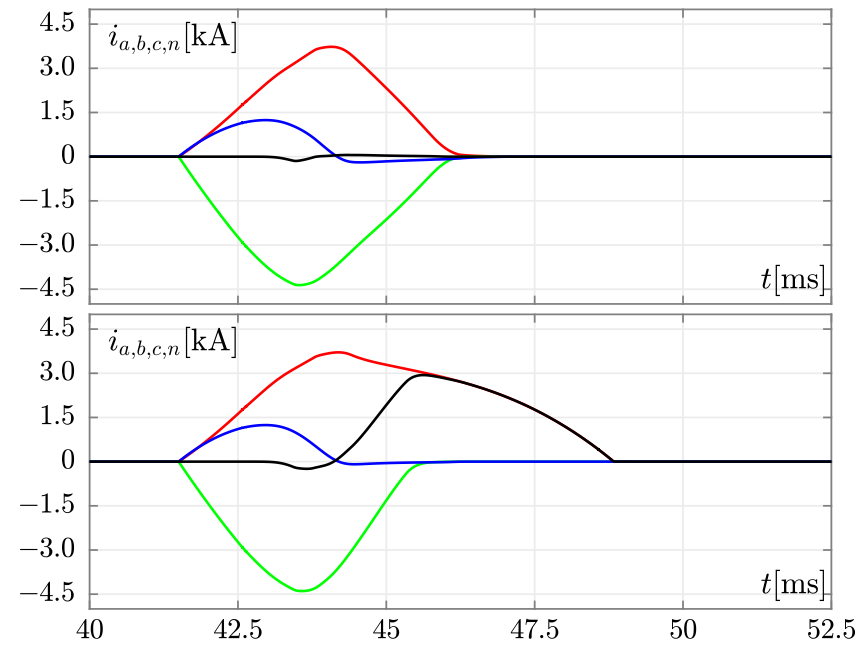

Fig. 12. The $i_{a}$ (red), $i_{b}$ (green), $i_{c}$ (blue) and $i_{n}$ (black) currents through the breaker during 4-poles MCB test. In the upper panel the delay in opening the neutral pole is null whereas in the lower panel is fixed at $6 \mathrm{~ms}$. $\mathrm{x}$-axis: time [ms], y-axis: current $[\mathrm{kA}]$. 
by the metallic constants to start their separation. It may be related to the contacts separation speed and surface roughness. It is not a critical parameter, since it also contributes to the numerical "trick" to ignite the arc. $x_{O}$ is the initial arc length when the contacts separate.

\section{REFERENCES}

[1] A. Balestrero and L. Ghezzi, "Modeling and simulation of low voltage arcs," Ph.D. dissertation, TU Delft, 2010, adv. Prof. L. van der Sluis.

[2] C. W. G. 13.01, "Applications of black box modelling to circuit breakers," Electra, no. 149, pp. 40-71, 1993.

[3] A. Balestrero, L. Ghezzi, M. Popov, G. Tribulato, and L. van der Sluis, "Black box modeling of low-voltage circuit breakers," IEEE Transactions on Power Delivery, vol. 25, no. 4, pp. 2481-2488, Oct 2010.

[4] O. Mayr, "Beiträge zur theorie des statischen und des dynamischen lichtbogens," Archiv für Elektrotechnik, vol. 37, no. 12, pp. 588-608, 1943.

[5] P. H. Schavemaker and L. van der Sluis, "An improved mayr-type arc model based on current-zero measurements [circuit breakers]," IEEE Transactions on Power Delivery, vol. 15, no. 2, pp. 580-584, Apr 2000.

[6] P. Schavemaker and L. van der Sluis, "The arc model blockset," in Proceedings of the Second Iasted International Conference Power and Energy Systems (EuroPES), Crete, Greece, June 2002.

[7] J. L. Guardado, S. G. Maximov, E. Melgoza, J. L. Naredo, and P. Moreno, "An improved arc model before current zero based on the combined mayr and cassie arc models," IEEE Transactions on Power Delivery, vol. 20, no. 1, pp. 138-142, Jan 2005.

[8] W. Rieder and J. Urbanek, "New aspects of current zero research on circuit breaker reignition. a theory of thermal non-equilibrium arc conditions," CIGRE Report, Paris, no. 107, 1966.

[9] J. Urbanek, "The time constant of high voltage circuit breaker arcs before current zero," Proceedings of the IEEE, vol. 59, no. 4, pp. 502508,1971

[10] F. Bizzarri and A. Brambilla, "Brushing up on the urbanek black box arc model," IEEE Transactions on Circuits and Systems I: Regular Papers, vol. 65 , no. 5, pp. 1675-1683, May 2018.

[11] F. Bizzarri, A. Brambilla, L. Ghezzi, and F. Rigamonti, "The urbanek black box arc model in passive resonance circuit breakers for hvdc applications," in 2018 IEEE International Symposium on Circuits and Systems, 2018, pp. 1-5.

[12] J. W. McBride and P. M. Weaver, "Review of arcing phenomena in low voltage current limiting circuit breakers," IEE Proceedings - Science, Measurement and Technology, vol. 148, no. 1, pp. 1-7, Jan 2001.

[13] F. Karetta and M. Lindmayer, "Simulation of the gasdynamic and electromagnetic processes in low voltage switching arcs," IEEE Transactions on Components, Packaging, and Manufacturing Technology: Part A, vol. 21, no. 1, pp. 96-103, Mar 1998.

[14] M. Lindmayer, E. Marzahn, A. Mutzke, T. Ruther, and M. Springstubbe, "The process of arc splitting between metal plates in low voltage arc chutes," IEEE Transactions on Components and Packaging Technologies, vol. 29, no. 2, pp. 310-317, June 2006.

[15] D. McKenzie and A. Ross, "Predator-prey dynamics stabilised by nonlinearity explain oscillations in dust-forming plasmas," Nature/Scientific Reports, vol. 6, no. 24040, April 2016.

[16] W. Rieder, Plasma und Lichtbogen, ser. Hochschul-Lehrbuch. Vieweg, 1967.

[17] Y. Naghizadeh-Kashani, Y. Cressault, and A. Gleizes, "Net emission coefficient of air thermal plasmas," Journal of Physics D: Applied Physics, vol. 35, no. 22, pp. 2925-2934, 2002.

[18] P. Antognetti, Ed., Semiconductor Device Modeling with SPICE. McGraw-Hill, Inc., 1988.

[19] M. Seeger, G. Naidis, A. Steffens, H. Nordborg, and M. Claessens, "Investigation of the dielectric recovery in synthetic air in a high voltage circuit breaker," Journal of Physics D: Applied Physics, vol. 38, no. 11, May 2005.

[20] M. Seeger, M. Schwinne, R. Bini, N. Mahdizadeh, and T. Votteler, "Dielectric recovery in a high-voltage circuit breaker in SF6," Journal of Physics D: Applied Physics, vol. 45, no. 39, 2012.

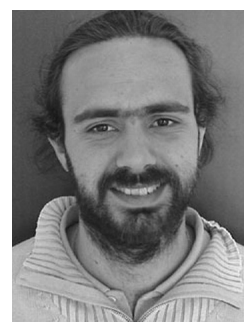

Federico Bizzarri (M'12-SM'14) was born in Genoa, Italy, in 1974. He received the Laurea (M.Sc.) five-year degree (summa cum laude) in electronic engineering and the Ph.D. degree in electrical engineering from the University of Genoa, Genoa, Italy, in 1998 and 2001, respectively.

Since June 2010 he has been a temporary research contract assistant professor at the Electronic and Information Department of the Politecnico di Milano, Milan, Italy. In 2000 he was a visitor to EPFL, Lausanne, Switzerland. From 2002 to 2008 he had been a post-doctoral research assistant in the Biophysical and Electronic Engineering Department of the University of Genova, Italy. In 2009 he was a post-doctoral research assistant in Advanced Research Center on Electronic Systems for Information and Communication Technologies "E. De Castro" (ARCES) at the University of Bologna, Italy.

His main research interests are in the area of nonlinear circuits, with emphasis on chaotic dynamics and bifurcation theory, circuit models of nonlinear systems, image processing, circuit theory and simulation. He is the author or coauthor of about 80 scientific papers, more than an half of which have been published in international journals. He is a research fellow of the Advanced Research Center on Electronic Systems for Information and Communication Technologies "E. De Castro" (ARCES) at the University of Bologna, Italy.

He served has as an Associate Editor of the IEEE Transactions on Circuits and Systems - Part I from 2012 to 2015 and he was awarded as one of the 2012-2013 Best Associate Editors of this journal. In 2103, 2015-2017, he has been a member of the Review Committee for the Nonlinear Circuits and Systems track at the IEEE International Symposium on Circuits and Systems.

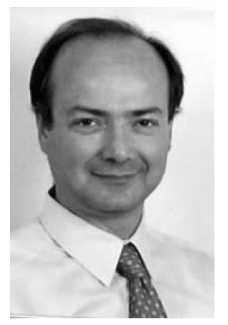

Angelo Brambilla (M'16) received the Dr. Ing. degree in electronics engineering from the University of Pavia, Pavia, Italy, in 1986. Currently he is full professor at the Dipartimento di Elettronica e Informazione, Politecnico di Milano, Milano, Italy, where he has been working in the areas of circuit analysis and simulation.

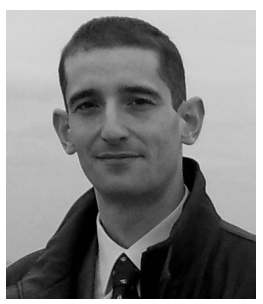

Luca Ghezzi received a M.Sc. in civil engineering from the Technical University (Politecnico) of Milano, Italy (1998), a M.Sc. in mathematics from the University of Milano, Italy (2007), and a Ph.D. in electrotechnology from the Technical University of Delft, the Netherlands (2010). He works for $\mathrm{ABB}$, involved in modeling and simulation of, a.o., electric arcs, approached both with multiphysics and at circuit level.

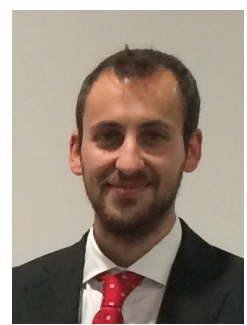

Francesco Rigamonti received the M.Sc. degree in nuclear physics from Universitá degli studi di Pavia (Pavia, Italy), working on nuclear photoreactions on polarized targets. In 2011 he joined ABB and, in parallel, in 2017 he received his executive Ph.D. in electrical engineering at Politecnico di Milano (Milan, Italy), focused on magnetic inverse problem applied to low voltage breakers. His work mainly regards physical modelling and computational aspects of low voltage electric arc. 